\title{
Cosmological viscous fluid models describing infinite time singularities in $f(T)$ gravity
}

\author{
R. D. Boko ${ }^{1,2, a}$, M. J. S. Houndjo ${ }^{1,2, b}$ \\ ${ }^{1}$ Faculté des Sciences et Techniques de Natitingou, UNSTIM, Abomey, Benin \\ ${ }^{2}$ Institut de Mathématiques et de Sciences Physiques (IMSP), 01 BP 613, Porto-Novo, Benin
}

Received: 18 April 2020 / Accepted: 16 July 2020 / Published online: 16 September 2020

(C) The Author(s) 2020

\begin{abstract}
In this paper we explore the state parameter behaviour of the interacting viscous dark energy in $f(T)$ gravity. Using constant deceleration parameter we investigate the cosmological implications of the viscosity and interaction between the dark components (energy and matter) in terms of Redshift. So doing, the viscosity and the interaction between the two fluids are parameterized by constants $\delta$ and $\xi$ respectively. In the later part of the paper, we explore some bulk viscosity models describing Little Rip and Pseudo Rip future singularities within $f(T)$ modified gravity. We obtain gravitational equations of motion for viscous dark energy coupled with dark matter. Solving these equations, we found analytic expressions for characteristic properties of these cosmological models.
\end{abstract}

\section{Introduction}

Since the accelerated phase of universe expansion has been discovered by cosmological measurements obtained from SNe Ia, WMAP, SDSS and X-ray [1-14], many cosmologists have done huge efforts to explain this strange phenomenon. An exotic fluid with a large negative pressure named dark fluid would be the origin of this accelerated expansion. So, many models have been proposed and explored as $\Lambda \mathrm{CDM}$ model which is the simplest model named cosmological constant. Besides this model, other models have been investigated (quintessence phantom, Tachyon, holographic dark energy, K-essence and Chaplyging gas) but unfortunately most of them do not permit to achieve the goal. According to the astrophysical observations, it has been mentioned that the cosmic medium is not a perfect fluid. So, various viscous fluids have been introduced to satisfy an inhomogeneous equation of state. Many authors have explored cosmo- logical viscous fluid effects on the dynamical evolution of the universe. Brevik et al. have considered inflation produced by two coupled fluids in the Friedmann-Robertson-Walker universe. They have used an inhomogeneous equation of state for the fluid to investigate different cosmological models for describing inflation [15]. Viscous Cosmology presenting various problems has been investigated by many authors [1632]. Some alternative theories like $f(T)$ and $f(R)$ expecting to explain accelerated expansion of the universe, have been explored in references [33-37]. Possible avoidance of finitetime singularities from viscosity in $f(T)$ gravity have been investigated [38]. In reference [39], authors have studied the interacting cosmological viscous fluid with infinite-time singularities solutions in $f(R)$ gravity and found interesting results. In this paper, we follow the same step but in area of modified $f(T)$ gravity. From the constant deceleration parameter $q$ we construct the scale factor that is expressed in terms of redshift. We then define the EoS for the dark energy in two cases. We first do not consider the interaction between viscous dark energy and dark matter, and secondly the interacting parameter is taking into account. We also study the bulk viscosity solutions from gravitational equation established from Freedmann-Robertson-Walker equations and viscous dark energy equation of state. We found that the bulk viscosity and the thermodynamic parameter present some corrections, in the EoS for the dark energy.

The paper is organised as follow: In Sect. 2, we present the formalism of $f(T)$ gravity and motion equations. In Sect. 3, we investigate the behavior of the evolution of universe filled with viscous dark energy coupled or not with dark matter. The Sect. 4 is devoted to analyse some bulk viscosity models of two future singularities (Little and Pseudo Rip) in the framework of $f(T)$ gravity. The paper is ended by a conclusion and remarks in Sect. 4.

\footnotetext{
a e-mail: docenzi@yahoo.fr (corresponding author)

be-mail: sthoundjo@yahoo.fr
} 


\section{Motion equations in $f(T)$ theory}

The modified theory of gravity based on the torsion scalar is the one for which the geometric part of the action is an algebraic function depending on the torsion. In the same way as in the Teleparallel gravity, the geometric elements are described using orthonormal tetrads components defined in the tangent space at each point of the manifold. In general the line element can be written as

$d s^{2}=g_{\mu \nu} d x^{\mu} d x^{\nu}=\eta_{i j} \theta^{i} \theta^{j}$,

where we define the following elements

$d x^{\mu}=e_{i}^{\mu} \theta^{i} \quad \theta^{i}=e_{\mu}^{i} d x^{\mu}$.

Note that $\eta_{i j}=\operatorname{diag}(1,-1,-1,-1)$ is the metric related to the Minkowskian spacetime and the $\left\{e_{\mu}^{i}\right\}$ are the components of the tetrad which satisfy the following identity

$e_{i}^{\mu} e^{i}{ }_{v}=\delta_{v}^{\mu}, \quad e_{\mu}^{i} e^{\mu}{ }_{j}=\delta_{j}^{i}$.

The connection used in this theory is the one of Weizenbock's, defined by

$\Gamma_{\mu \nu}^{\lambda}=e_{i}{ }^{\lambda} \partial_{\mu} e^{i}{ }_{\nu}=-e^{i}{ }_{\mu} \partial_{\nu} e_{i}^{\lambda}$.

Once the previous connection assumed, one can then express the main geometric objects; the torsion tensor's components as

$T_{\mu \nu}^{\lambda}=\Gamma_{\mu \nu}^{\lambda}-\Gamma_{\nu \mu}^{\lambda}$,

which is used in the definition of the contorsion tensor as

$K_{\lambda}^{\mu \nu}=-\frac{1}{2}\left(T_{\lambda}^{\mu \nu}-T_{\lambda}^{v \mu}+T_{\lambda}^{\nu \mu}\right)$.

The above objects (torsion and contorsion) are used to define a new tensor $S_{\lambda}{ }^{\mu v}$ as

$S_{\lambda}^{\mu \nu}=\frac{1}{2}\left(K_{\lambda}^{\mu \nu}+\delta_{\lambda}^{\mu} T_{\alpha}^{\alpha \nu}-\delta_{\lambda}^{\nu} T_{\alpha}^{\alpha \mu}\right)$.

The torsion scalar is defined from the previous tensor and the torsion tensor as

$T=T_{\mu \nu}^{\lambda} S_{\lambda}^{\mu \nu}$

Let's write the action for the modified $f(T)$ theory with matter as follows

$S=\int d^{4} x e\left[\frac{T+f(T)}{2 \kappa^{2}}+\mathcal{L}_{(\text {matter })}\right]$,

where $e \equiv \operatorname{det}\left[e_{\mu}^{i}\right]=\sqrt{-g}$ denotes the determinant of the tetrad, and $g$ the determinant of the space-time metric and $f(T)$, the algebraic function of $T$ torsion.

Making the functional variation of the action (9) with respect to the tetrads, we get the following equations of motion

$$
\begin{aligned}
& S_{\mu}{ }^{\nu \rho} \partial_{\rho} T f_{T T}+\left[e^{-1} e^{i}{ }_{\mu} \partial_{\rho}\left(e e_{i}{ }^{\mu} S_{\alpha}{ }^{\nu \lambda}\right)+T_{\lambda \mu}^{\alpha} S_{\alpha}{ }^{\nu \lambda}\right] \\
& \quad \times f_{T}+\frac{1}{4} \delta_{\mu}^{\nu} f=\frac{\kappa^{2}}{2} \mathcal{T}_{\mu}^{\nu},
\end{aligned}
$$

where $\mathcal{T}_{\mu}^{v}$ denotes the stress tensor such that

$\mathcal{T}_{\mu}^{v}=(\rho+p) u_{\mu} u^{\nu}-p \delta_{\mu}^{v}$,

$\rho$ and $p$ being the energy density and the pressure of ordinary content of the universe respectively and $u^{\mu}$, the fourvelocity such that $u^{\mu} u_{\mu}=1$. Still with the considered signature $(+,-,-,-)$, we assume the line element for the flat Robertson-Walker universe as

$d S^{2}=d t^{2}-a^{2}(t) d \mathbf{x}^{2}$

where $a(t)$ is the universe scalar factor. Therefore, the torsion scalar is performed gives:

$T=-6 H^{2}$.

From (10) to (12), one gets the following gravitational field equations:

$$
\begin{aligned}
& 6 H^{2}+12 H^{2} f_{T}+f=2 \kappa^{2} \rho, \\
& 2\left(2 \dot{H}+3 H^{2}\right)+f+4\left(\dot{H}+3 H^{2}\right) f_{T} \\
& \quad-48 H^{2} \dot{H} f_{T T}=-2 \kappa^{2} p .
\end{aligned}
$$

Here the "dot" denotes the derivative with respect to the cosmic time $t, H=\dot{a}(t) / a(t)$, the Hubble parameter and $\rho$ and $p$, the matter energy-density and pressure, respectively. $f_{T}$ and $f_{T T}$ are the derivatives of $f(T)$ with respect of torsion $T$ respectively. By assuming that $\kappa^{2}=1$, the above Eqs. (14), (15) are written as:

$$
\begin{aligned}
3 H^{2} & =-6 H^{2} f_{T}-\frac{1}{2} f+\rho, \\
-2 \dot{H}-3 H^{2} & =\frac{1}{2} f+2\left(\dot{H}+3 H^{2}\right) f_{T}+2 H \dot{f}_{T}+p .
\end{aligned}
$$

Since the effective energy density and effective pressure take the form:

$$
\begin{aligned}
3 H^{2} & =\rho_{e f f}, \\
-2 \dot{H}-3 H^{2} & =p_{e f f},
\end{aligned}
$$

the energy density and pressure of dark energy are defined as:

$$
\begin{aligned}
& \rho_{D E}=-6 H^{2} f_{T}-\frac{1}{2} f, \\
& p_{D E}=\frac{1}{2} f+2\left(\dot{H}+3 H^{2}\right) f_{T}+2 H \dot{f}_{T} .
\end{aligned}
$$




\section{Dynamical evolution of the universe filled with dark fluids}

In Ref. [39], the authors have used viable $f(R)$-gravity representing a realistic scenario for the dark energy epoch. This model describes the late-time cosmological evolution of the Universe filled with dark matter. Many authors have studied this model and found interesting results [40-42]. In $f(T)$ modified gravity, power-law models have been explored and reconstructed describing the evolution scenarios of Universe [43-46]. In the following, we will consider $f(T)$ power-law model to investigate Universe dynamics by using constant deceleration parameter.

$f(T)=e(-T)^{h}$

where $e=\frac{1-\Omega_{m o}}{2 n-1}\left(6 H_{o}^{2}\right)^{(1-n)}$ and $h$ are parameters. From the decceleration parameter $q=-\frac{a \ddot{a}}{\dot{a}^{2}}$, the scale factor take the form $[47,48]$ :

$a(t)=\left[(1+q)\left(\frac{1}{k} t+l\right)\right]^{\frac{1}{1+q}}$

where $q$ is assumed to be a constant deceleration parameter. $k$ and $l$ are constants of integration. In terms of redshift, this scale factor takes the form:

$a(t)=\frac{1}{1+z}, \quad$ with $\quad z=\frac{1}{\left[(1+q)\left(\frac{1}{k} t+l\right)\right]^{\frac{1}{1+q}}}-1$

We now assume the dark fluid equation of state in the following form $[39,49]$ :

$\tilde{P}_{D E}=p_{D E}-3 H \delta\left(\rho_{D E}\right)$

where $\delta\left(\rho_{D E}\right)$ is the bulk viscosity depending on $\rho_{D E}$ in general way. Acording to the thermodynamical grounds and in conventional physics, $\delta\left(\rho_{D E}\right)$ must be positive, thus the positive sign of the entropy changes and an irreversible process would be guarantee [39]. In the following, the dark matter will be assumed to be a dust, so $\omega_{m}=0$. We will also consider in the first party of this work, the general form of bulk viscosity as [53] $\delta\left(\rho_{D E}\right)=\delta_{o}\left(\rho_{D E}\right)^{\eta}$, where $\delta_{o}>0$ and $\eta$ are constant parameters (Figs. 1, 2).

\subsection{Non-coupled dark fluids}

The dark energy continuity equation is defined as:

$\dot{\rho}_{D E}+3 H\left(\rho_{D E}+p_{D E}\right)=9 H^{2} \delta_{o}^{\eta}\left(\rho_{D E}\right)$,

with $p_{D E}=\omega_{D E} \rho_{D E}$. By Using (20) and taking into acount (22) and (23), we find the dark energy density as:

$\rho_{D E}(t)=e \cdot 6^{h}\left(h-\frac{1}{2}\right)\left[\frac{1}{k(1+q)} \cdot \frac{1}{\left(\frac{1}{k} t+l\right)}\right]^{2 h}$,

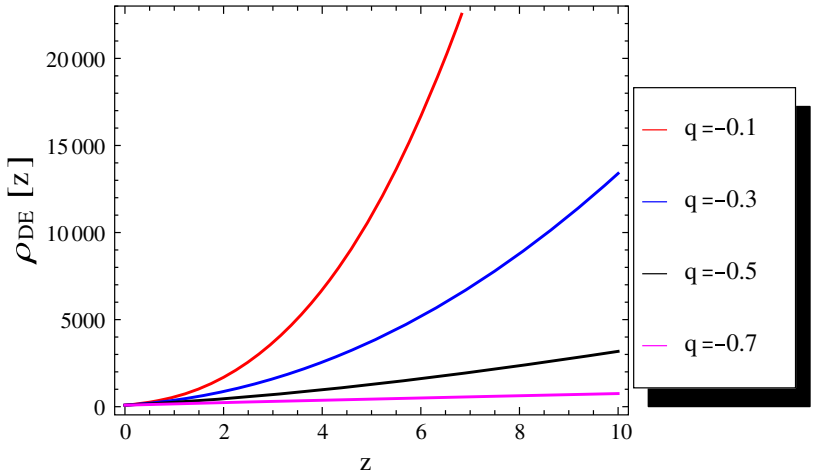

Fig. 1 The variations of energy density $\rho_{D E}$ of Dark energy is depicted in the graph. The graphs are plotted for $h=3 / 2, k=3 / 4, e=5 / 2$

with 24 , this dark energy density in terms of redshift, takes the form:

$\rho_{D E}(z)=\frac{e .6^{h}\left(h-\frac{1}{2}\right)}{k^{2 h}}(z+1)^{2 h(1+q)}$.

By following the same steps as previously, we from (21) to (23), write the dark energy pressure in the form:

$$
\begin{aligned}
\tilde{P}_{D E}(t)= & \frac{1}{6}\left\{A k^{-2 h}\left[(1+q)\left(\frac{1}{k} t+l\right)\right]^{-2 h}\right. \\
& \left.-\frac{18 \delta_{o}^{\eta}}{k}\left[(1+q)\left(\frac{1}{k} t+l\right)\right]^{-1}\right\},
\end{aligned}
$$

we also express the dark energy pressure in terms of redshift, that is:

$\tilde{P}_{D E}(z)=\frac{1}{6}\left[A k^{-2 h}(z+1)^{2 h(1+q)}-\frac{18 \delta_{o}^{\eta}}{k}(z+1)^{(1+q)}\right]$,

with $A=6^{h}\left[12^{h}(-2+q)+e\left(3-4 h(1+q)+4 h^{2}(1+q)\right)\right]$. Let's now plot the functions (28), and (30).

One see that, the energy density increases as the deceleration parameter diminishes with increasing Redshift. For $q=-1$, the energy density of dark energy increases indefinitely. Note that for particular value of $h=1 / 2$, this energy is null.

In the graph of left hand-side, the pressure increases as $q$ decreases, but in the ones of right hand-side, the pressure evolves in the same way as the viscosity parameter (Fig. 3).

We now, from the Eqs. (26) and (27) define the EoS parameter of dark energy $W_{D E e f f}=\frac{\tilde{P}_{D E}(t)}{\rho(t)}$ :

$$
\begin{aligned}
& W_{\text {DEeff }}(t)=\frac{1}{e \cdot 6^{1+h}\left(h-\frac{1}{2}\right)}\left[\frac{1}{(1+q)} \cdot \frac{1}{\left(\frac{1}{k} t+l\right)}\right]^{-2 h} \\
& \times\left\{A k^{-2 h}\left[(1+q)\left(\frac{1}{k} t+l\right)\right]^{-2 h}-\frac{18 \delta_{o}^{\eta}}{(1+q)(k l+t)}\right\},
\end{aligned}
$$



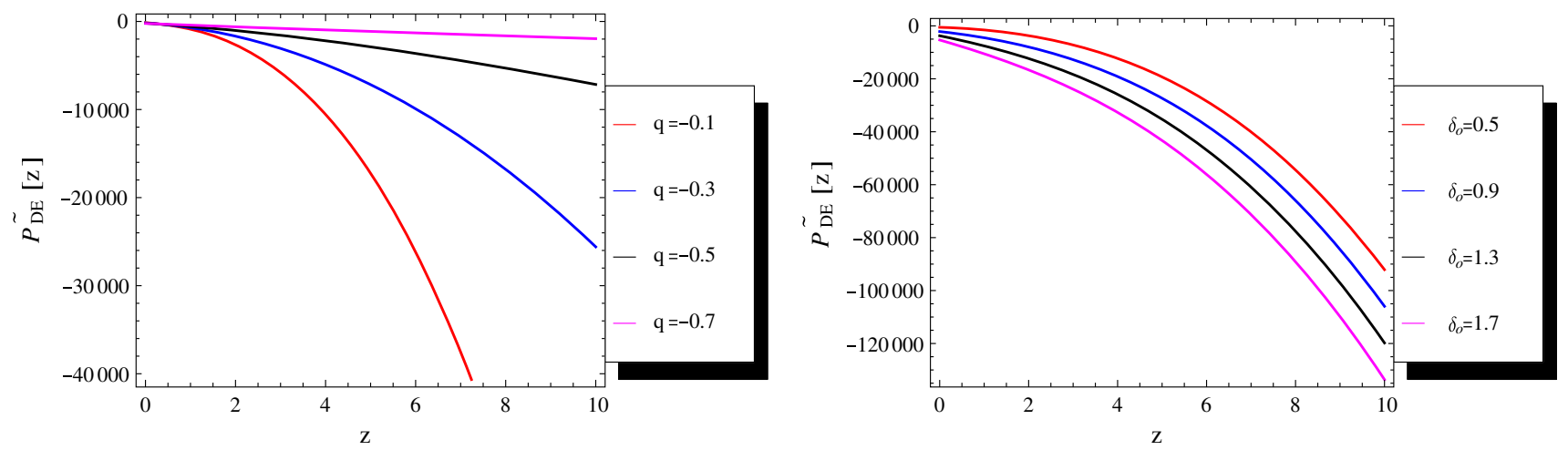

Fig. 2 The pressure $\tilde{P}_{D E}$ of Dark energy are plotted at left hand-side for variable deceleration parameter $q$ and at right hand-side for variable bulk viscosity parameter $\delta_{o}$. The graphs are plotted for $\eta=1, h=3 / 2, k=3 / 4, e=5 / 2, q=-0.1$ and $\delta_{o}=1 / 2$ (panel in left hand-side)

that in terms of redshift leads to:

$$
\begin{aligned}
W_{\text {DEeff }}(z)= & \frac{(z+1)^{-2 h(1+q)}}{e \cdot 6^{1+h}\left(h-\frac{1}{2}\right)} \\
& \times\left[A k^{-2 h}(z+1)^{2 h(1+q)}-18 \delta_{o}^{\eta}(z+1)^{1+q}\right] .
\end{aligned}
$$

The variations of EoS $W_{D E e f f}$ for dark energy are depicted in the above graphs. At the left hand-side, the EoS parameter is plotted for fixed value of viscosity parameter $\delta_{o}$ and variable values of deceleration parameter $q$. But at the right hand-side, one sees the function $W_{D E e f f}$ plotted for fixed value of $q$ and variable values of $\delta_{o}$. The variations of $W_{D E \text { eff }}$ are plotted for $\eta=1, h=3 / 2, k=3 / 4, e=5 / 2$, $q=-0.1, \delta_{o}=2.5$ (panel in left hand-side)

We can see that these both EoS parameters evolve in quintessence phase, then in $f(T)$ gravity, universe is in accelerated expansion phase with high and low redshift. In [53], the EoS parameter for viscous Dark Energy varies in unstable phantom region and there is a transition from phantom to the cosmological constant phase at late time. In $f(R)$ modified gravity [39], as we approach null redshift, universe seems to enter phantom phase. The left hand graph, shows that, at particular point $(4,-0.23)$, the EoS parameter of DE seems not to be dependent of deceleration parameter $q$.

\subsection{Coupled dark fluids}

In this section, we consider an interaction between the dark energy and dark matter and the corresponding continuity equations are written as:

$$
\left\{\begin{array}{l}
\dot{\rho}_{D E}+3 H\left(\rho_{D E}+\tilde{P}_{D E}\right)=-Q \\
\dot{\rho}+3 H(\rho+p)=Q
\end{array}\right.
$$

One expresses the interaction between the both dark fluids by the quantity $Q$ (Fig. 4). In order to ensure that the second law of thermodynamics is fulfilled, the interaction parameter $Q$ must be positive [50-52]. In the same way like [53], one writes the interaction parameter in the form:

$Q=H \xi \rho$,

where $\xi$ is an interaction constant. From (33), (34), one obtains:

$\rho=\rho_{o}\left(\frac{a}{a_{o}}\right)^{\xi-3(1+\omega)}$,

and in terms of redshift, it takes the form:

$\rho(z)=\rho_{o}\left(\frac{1+z}{1+z_{o}}\right)^{3(1+\omega)-\xi}$.

The Eqs. (27) and (36) permit to obtain the EoS parameter for interacting dark energy as:

$$
\begin{aligned}
& W_{D E e f f}(t) \\
& =\frac{A k^{-2 h}\left[(1+q)\left(\frac{1}{k} t+l\right)\right]^{-2 h}-\frac{18 \delta_{0}^{\eta}}{(1+q)(k l+t)}}{6\left[\frac{3}{[(1+q)(k l+t)]^{2}}-\left(\frac{1}{(1+q)(k l+t)}\right)^{\frac{\xi-3(1+\omega)}{1+q}} \rho_{o} a_{o}^{-\xi+3(1+\omega)}\right]},
\end{aligned}
$$

and the other expression of this dark energy EoS parameter in terms of redshift is written as:

$$
\begin{aligned}
& W_{D E e f f}(z) \\
& =\frac{A k^{-2 h}(z+1)^{2 h(1+q)}-\frac{18 \delta_{o}^{\eta}}{k}(z+1)^{(1+q)}}{6\left[\frac{3}{k^{2}}(z+1)^{2(1+q)}-\left(\frac{(z+1)^{(1+q)}}{k}\right)^{\frac{\xi-3(1+\omega)}{1+q}} \rho_{o} a_{o}^{-\xi+3(1+\omega)}\right]} .
\end{aligned}
$$

In the left panel, one sees that with interaction parameter, the EoS of both non-viscous and viscous dark energy approach the cosmological constant region $\left(W_{D E e f f}=-1\right)$ for low values of redshift. But in the panel at the right handside, the EoS parameter has the same behavior as the one in [53] because a comparative analysis of these both panels shows that the parameter $W_{D E e f f}$ of non-viscous dark energy 

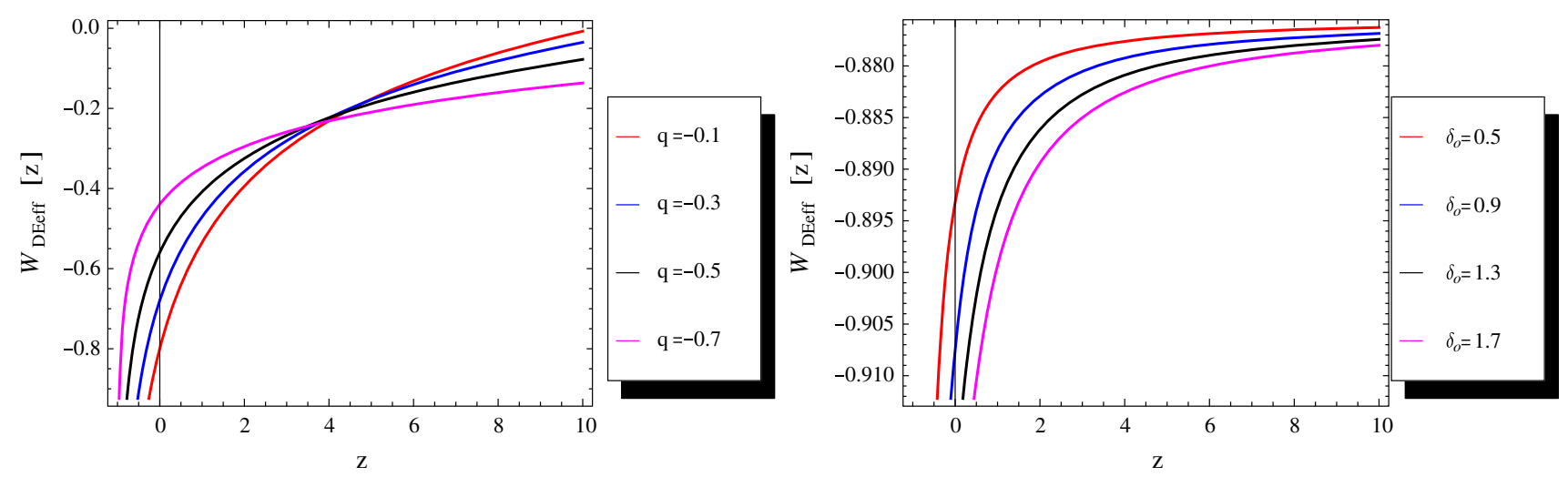

Fig. 3 The variations of EoS $W_{D E e f f}$ for dark energy is depicted in the above graphs

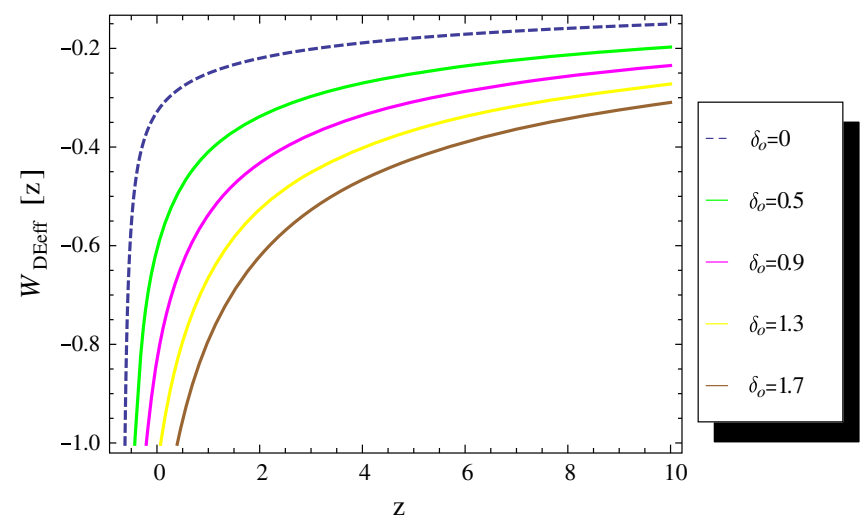

Fig. 4 The variations of EoS parameter $W_{D E e f f}$ of viscous dark energy for fixed value of $\xi$ and for $\delta_{o}$ are respectively depicted in the graph at left-hand side and in the graph at right-hand side. The graphs are plotted

$\left(\delta_{o}=0\right)$ is only varying in quintessence region whereas with high interaction $(\xi>2) W_{D E e f f}$ starts from quintessence region, crossing the phantom divided line (PDL), and evolves to phantom region. The opposite phenomenon is observed in $f(R)$ gravity [39], where the interaction alleviates the EoS parameter of dark energy from phantom region (for high redshift) to quintessence region (for low redshift) that evolves to the phantom region.

\section{Viscous fluids models describing future singularities}

We investigate in this area, the gravitational equation of motion that gives some bulk viscosity models describing some future singularities. Here, we are interested by Little Rip and the Pseudo Rip future singularities.

\subsection{Little Rip}

The characteristic of Little Rip singularity is an increasing energy density $\rho$ with time, that needs an infinite time to

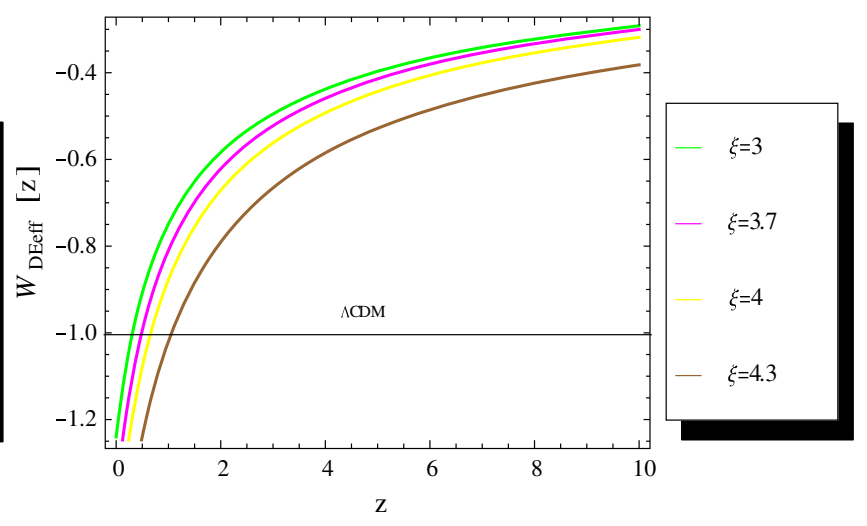

for $\eta=1, h=3 / 2, k=3 / 4, e=5 / 2, q=-0.1, \rho_{o}=0.9, a_{o}=1$, $\omega=0, \xi=2$ and $\delta_{o}=1.5$ (panel in right hand-side)

appear. Thus, in this case, the EoS parameter $\omega<-1$, and $\omega \rightarrow-1$ asymptotically [54].

Let's consider the Hubble parameter increasing exponentially with time [50]

$H(t)=H_{o} \exp (\gamma t)$

$\gamma$ and $H_{o}$ (Hubble parameter at present time) are positive constants. Assuming that the dark matter is the dust $(p=0)$, the Eq. (33) gives:

$\rho(H)=\rho_{o} \exp \left(\frac{3-\delta}{\gamma} H_{o}\right) \exp \left(\frac{\delta-3}{\gamma} H\right)$,

with $\rho_{o}$ an integration constant. For $\delta<3$, it happens that $\rho \rightarrow 0$ when $t \rightarrow \infty$. Considering the relations (18), (25) and the Eq. (33), one finds the gravitational equation of motion for viscous dark energy coupled with dark matter as:

$6 \dot{H} H-\dot{\rho}+3 H\left(2 \dot{H} f_{T}+2 H \dot{f}_{T}-3 H \delta\right)=-H \xi \rho$. 
With (41) and Eq. (21), one obtains the Hubble-dependent bulk viscosity that is expressed by:

$$
\begin{aligned}
\delta(H)= & \frac{1}{9 H}[(2 \xi-3) \rho+6 \gamma H \\
& \left.+6(-2 h+3) \gamma \operatorname{eh} H\left(6 H^{2}\right)^{h-1}\right] .
\end{aligned}
$$

When $t \rightarrow \infty$, then $H \rightarrow \infty$ and $\delta \rightarrow \infty$ if $h>1$ or the bulk viscosity tends to finite value if $h<1$.

We now, consider another expression of the Little Rip model where the Hubble parameter is a double exponentially increasing with time:

$H=H_{o} \exp \left(B e^{(\gamma t)}\right)$,

with $H_{O}, D$ and $\gamma$ are positive constants.

Using the Hubble parameter (43) and by solving the gravitational equation of motion (33) for dark matter, we find:

$\rho(t)=\rho_{o} \exp \left[(\xi-3) \gamma H \ln \left(\frac{H}{H_{o}}\right)\right]$

with $\rho_{o}$ the constant of integration (Fig. 5).

Thus, we find from the gravitational equation of motion (41), for dark energy:

$$
\begin{aligned}
\delta(H)= & \frac{1}{9 H}\{(2 \xi-3) \rho+6 \gamma H \\
& \times\left[1+e h\left(6 H^{2}\right)^{h-1}+12 h e H^{2}(h-1)\left(6 H^{2}\right)^{h-2}\right] \\
& \left.\times \ln \left(\frac{H}{H_{o}}\right)\right\} .
\end{aligned}
$$

\subsection{Pseudo Rip}

In the Pseudo Rip future singularity case, the Hubble parameter tends to cosmological constant as $t \rightarrow \infty$. Thus, a de Sitter space is approached by the universe. Let's now analyse that model throughout the analysis of the Litte Rip model.

We first assume that the Hubble parameter takes the form:

$H=H_{o}-H_{1} \exp (-\lambda t)$,

where $H_{o}, H_{1}$ and $\lambda$ are the positive constants, $H_{1}<H_{o}$ with $t>0$. This means that $H \rightarrow H_{o}$ in the late-time universe.

By solving the gravitational equation of motion (33) and using the Hubble parameter (49) we obtain:

$\rho(t)=\rho_{o} \exp \left[(\delta-3)\left(H_{o} t-\frac{H-H_{o}}{\lambda}\right)\right]$,

with $\rho_{o}$ an integration constant.

Using the dark matter density (40) with the power-law $f(T)$ model (22) and solving the gravitational (41), the bulk viscosity is written as

$$
\begin{aligned}
\delta(H)= & \frac{1}{9 H}\left\{(2 \xi-3) \rho+6 \lambda\left(H_{o}-H\right)\right. \\
& \left.\times\left[1+e h\left(6 H^{2}\right)^{h-1}+12 h e H^{2}(h-1)\left(6 H^{2}\right)^{h-2}\right]\right\} .
\end{aligned}
$$

As the time evolves till the infinity, the Hubble parameter tends to the constant value $\left(H_{O}\right)$, and the viscosity parameter depends on the energy density that evolves in terms of parameter $\delta$ i.e $: \rho \rightarrow o$ if $\delta<3$ or $\rho \rightarrow \infty$ if $\delta>3$. Thus, $\delta(H) \rightarrow o$ for $\delta<3$ and $\delta(H) \rightarrow \infty$ for $\delta>3$ as the times evolves.

At the end of this section, we will explore a second example of cosmological model where the Hubble parameter is given by [39-56]:

$H=\frac{x_{r}}{\sqrt{3}}\left[1-\left(1-\frac{x_{o}}{x_{r}}\right) \exp \left(-\frac{\tilde{\gamma} \sqrt{3}}{2 x_{r}} t\right)\right]$

In the above expression, $x_{o}=\sqrt{\rho_{o}}$ is the present energy, $x_{r}$ is a finite, and $\tilde{\gamma}$ is a positive constant. As $t \rightarrow 0$, one have $H \rightarrow x_{o} / \sqrt{3}$. Note that, in the late-time universe $H \rightarrow$ $x_{r} / \sqrt{3}$, and the Hubble parameter tends asymptotically to the de Sitter solution.

The continuity Eq. (33) leads to the energy density of dark matter that is:

$$
\begin{aligned}
\rho(H, t)= & \rho_{o} \exp \left[\frac{2 x_{r}^{2}}{3 \tilde{\gamma}}(3-\xi)\left(1-\frac{\sqrt{3} H_{o}}{x_{r}}\right)\right] \\
& \times \exp \left\{(\xi-3) \frac{x_{r}}{\sqrt{3}}\left[t+\frac{2 x_{r}}{\tilde{\gamma} \sqrt{3}}\left(1-\frac{\sqrt{3}}{x_{r}} H\right)\right]\right\},
\end{aligned}
$$

where $\rho_{o}$ is an integration constant. As $t \rightarrow \infty$, one have $\rho \rightarrow \infty$ for $\xi>3$ but $\rho \rightarrow o$ for $\xi<3$.

Finally, the following bulk viscosity takes the form:

$$
\begin{aligned}
\delta(H)= & \frac{1}{9 H}\left\{(2 \xi-3) \rho+\frac{6 x_{r}}{\sqrt{3}}\left[1+e h\left(6 H^{2}\right)^{h-1}\right.\right. \\
& \left.\left.+12 h e H(h-1)\left(6 H^{2}\right)^{h-2}\right] \frac{\tilde{\gamma} \sqrt{3}}{2 x_{r}}\left(1-\frac{\sqrt{3}}{x_{r}} H\right)\right\} .
\end{aligned}
$$

Also we noticed that when $t \rightarrow \infty$ with $\xi<3$, one will have $\delta(H)$ be vanished but if $\xi>3$, it happens that $\delta(H) \rightarrow \infty$. Some corrections have appeared in the bulk viscosity due to the modified gravity model and the interaction between dark energy and dark matter (Fig. 6).

\section{Conclusion}

We have investigated in this work, cosmological behavior of universe filled with inhomogeneous dark energy coupled or not with dark matter in $f(T)$ modified gravity. It has also 


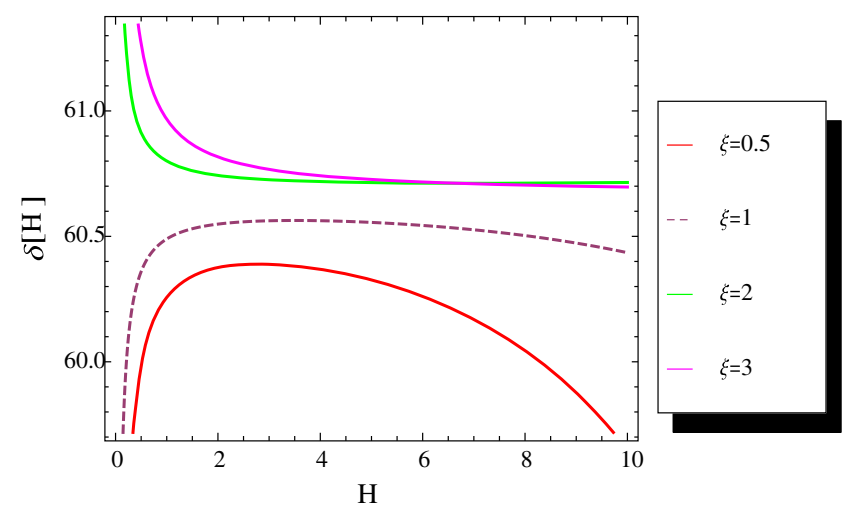

Fig. 5 We are illustrated through these graphs the behavior of $\delta$ versus the Hubble parameter. The first and second model of viscosity parameter are depicted in the both graphs for fixed values $\gamma=7, h=1$, $H_{o}=1, e=12, \rho_{o}=0.9$. In the graph at the left hand-side, we can see that at the beginning, the bulk viscosity increases (for low interact-

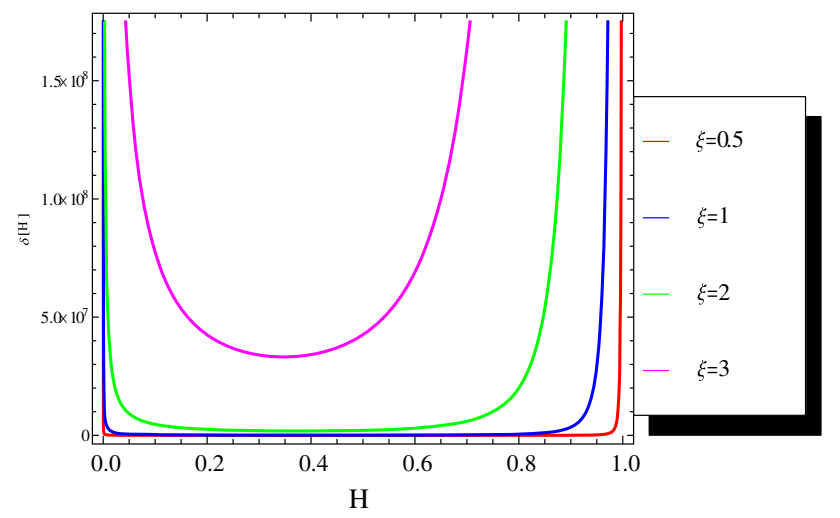

Fig. 6 The variations of bulk viscosity for Pseudo Rip singularity are plotted in the graph at the left hand-side (for first model) and in the graph at the right hand-side (for second model) respectively for the fixed values $\lambda=5, H_{o}=1, h=2, e=12, \rho_{o}=0.9$ and $\lambda=5, H_{o}=1$,

been studied, the bulk viscosity evolution describing future singularities throughout bounce cosmology. The first part of this work is devoted to analyse the dynamical evolution of EoS dark energy parameter that shows an universe in accelerated expansion. But with the strong interaction between the dark components, the universe evolves from the quintessence region to the phantomic ones for decreasing redshift. Note that in this case, the low interaction makes the universe only be in accelerated expansion phase as the time evolves. In comparison with [39], one note that with the lagragian $f(T)$ in power-law model, the results found are the same as the ones of [53]. In the second part of this paper, we investigate some bulk viscosity models solutions from gravitational equation that describe Little Rip and Pseudo Rip singularities in the framework of $f(T)$ gravity. This gravitational equation is a generalisation of that one found in [56]. We notice some corrections in the bulk viscosity and in the EoS parameter of dark

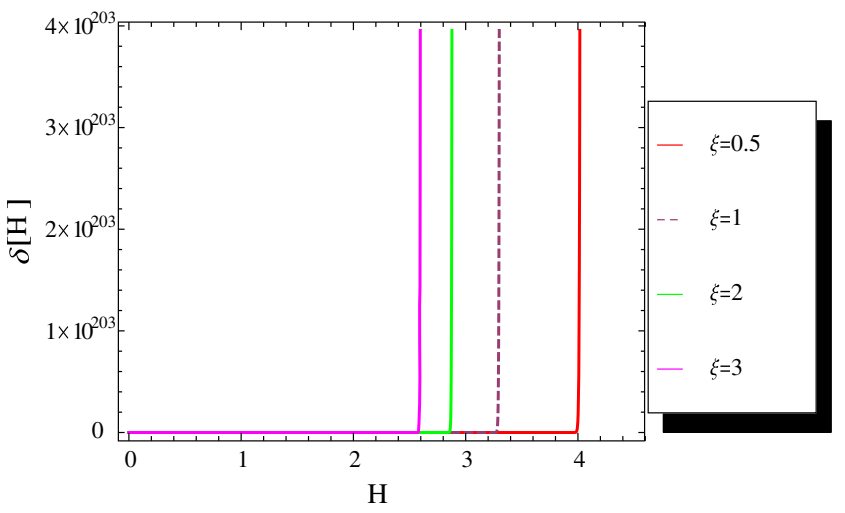

ing parameter), but decreases (for high $\xi$ ) and converges to the constant values as the time evolves indefinitely. Note that for $\xi<1, \delta \rightarrow 0$. whereas in the graph at the right hand-side, $\delta$ is nul at the beginning but diverges as the time increases

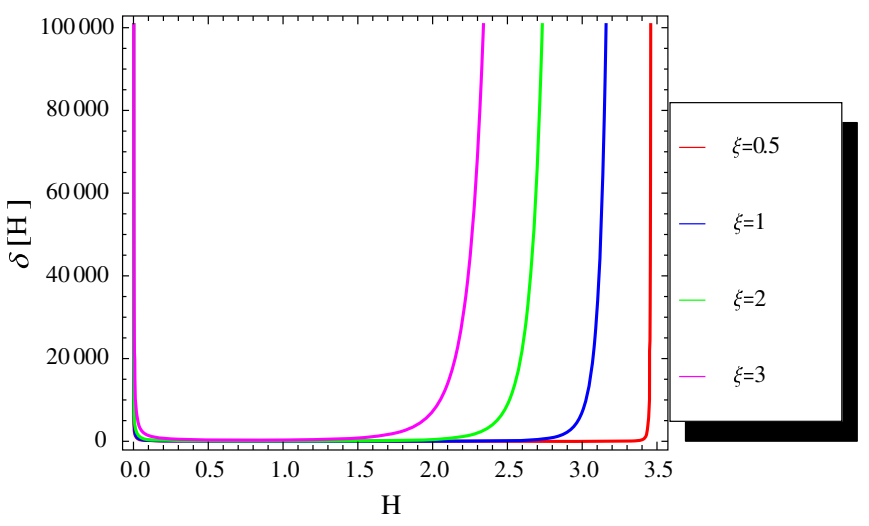

$h=2, e=12, \rho_{o}=0.9, x_{r}=6, \tilde{\gamma}=10$. In these graphs, we can see that, the viscosity decreases at the beginning and becomes null as the time evolves but diverges for increasing time. Note that, the viscosity is not null for high interaction parameter $\xi$

energy due to the $f(T)$ modified gravity and the coupling parameter. The analysis of the Little Rip bulk viscosity solutions shows that it could vanish for low interacting parameter $(\xi)$ and converge for high value of $\xi$. In Pseudo Rip case, these solutions decrease at the beginning and increase at late time.

Data Availability Statement This manuscript has associated data in a data repository. [Authors' comment: The data that support the findings of this study are available from the corresponding author, R. D. B., upon reasonable request].

Open Access This article is licensed under a Creative Commons Attribution 4.0 International License, which permits use, sharing, adaptation, distribution and reproduction in any medium or format, as long as you give appropriate credit to the original author(s) and the source, provide a link to the Creative Commons licence, and indicate if changes were made. The images or other third party material in this article are included in the article's Creative Commons licence, unless indicated otherwise in a credit line to the material. If material is not included in the article's Creative Commons licence and your intended 
use is not permitted by statutory regulation or exceeds the permitted use, you will need to obtain permission directly from the copyright holder. To view a copy of this licence, visit http://creativecomm ons.org/licenses/by/4.0/.

Funded by SCOAP ${ }^{3}$.

\section{References}

1. D.N. Spergel et al. [WMAP Collaboration], ApJS 170, 377 (2007)

2. L. Page et al. [WMAP Collaboration]. ApJS 170, 335 (2007)

3. G. Hinshaw et al. [WMAP Collaboration], ApJS 170, 288 (2007)

4. N. Jarosik et al. [WMAP Collaboration], ApJS 170, 263 (2007)

5. A.G. Riess et al. [Supernova Search Team Collaboration], Observational evidence from supernovae for an accelerating universe and a cosmological constant. Astrophys. J. Suppl. Ser. 116, 1009-1038 (1998)

6. S.J. Perlmutter et al., Discovery of a supernova explosion at half the age of the universe. Nature 391, 51-54 (1998). https://doi.org/ $10.1038 / 34124$

7. M.Tegmark et al., Cosmological parameters from SDSS and WMAP. Phys. Rev. D 69, Article ID: 103501 (2004). https://doi. org/10.1103/PhysRevD.69.103501

8. S. Perlmutter et al., Nature (London) 391, 51 (1998)

9. R. Knop et al., Astrophys. J. 598, 102 (2003)

10. A.G. Riess et al., Astrophys. J. 607, 665 (2004)

11. H. Jassal, J. Bagla, T. Padmanabhan, Phys. Rev. D 72, 103503 (2005)

12. S.W. Allen, R.W. Schmidt, H. Ebeling, A.C. Fabian, L. Van Speybroeck, Constraints on dark energy from chandra observations of the largest relaxed galaxy clusters. Mon. Not. R. Astron. Soc. 353, 457-467 (2004). https://doi.org/10.1111/j.1365-2966.2004. 08080.x

13. D.N. Spergel, L. Verde, H.V. Peiris, E. Komatsu, M.R. Nolta, C.L. Bennett, M. Halpern, G. Hinshaw, N. Jarosik, A. Kogut, M. Limon, S.S. Meyer, L. Page, G.S. Tucker, J.L. Weiland, E. Wollack, E.L. Wright, First year Wilkinson microwave anisotropy probe (WMAP) observations: determination of cosmological parameters. Astrophys. J. Suppl. Ser. 148, 175-194 (2003). (10.1086/)

14. E. Komatsu et al., Five-year Wilkinson microwave anisotropy probe (WMAP) observations: cosmological interpretation. Astrophys. J. Suppl. (2009)

15. I. Brevik, A.V. Timoshkin, Viscous coupled fluids in inflationary cosmology. arXiv:1509.06995v3

16. S. Weinberg, Gravitation and Cosmology: Principles and Applications of the General Theory of Relativity (Wiley, New York, 1972)

17. T. Padmanabham, S.M. Chitre, Phys. Lett. A 120, 433 (1977)

18. Ø. Grøn, Astrophys. Space Sci. 173, 191 (1990)

19. I. Brevik, L.T. Heen, Astrophys. Space Sci. 219, 99 (1994)

20. I. Brevik, A. Hallanger, Phys. Rev. D 69, 024009 (2004)

21. M. Cataldo, N. Cruz, S. Lepe, Phys. Lett. B 619, 5 (2005)

22. I. Brevik, J.M. Børven, S. Ng, Gen. Relativ. Gravit. 38, 907 (2006)

23. I. Brevik, S.D. Odintsov, Phys. Rev. D 65(067302), 067302 (2002). arXiv:gr-qc/0110105

24. I. Brevik, S. Nojiri, S.D. Odintsov, D. Saez-Gomez, Eur. Phys. J. C 69, 563 (2010). arXiv: 1002.1942

25. I. Brevik, O. Gorbunova, Gen. Relativ. Gravit. 37, 2039 (2005)

26. B. Li, J.D. Barrow, Phys. Rev. D 79, 103521 (2009)

27. I. Brevik, O. Gorbunova, D. Saez-Gomez, Gen. Relatiiv. Gravit. 42, 1513 (2010)

28. L. Sebastiani, Eur. Phys. J. C 69, 547 (2010). arXiv:1006.1610v3 [gr-qc]

29. H. Velten, D.J. Schwarz, Phys. Rev. D 86, 083501 (2012)

30. H. Velten, D.J. Schwarz, J.C. Fabris, W. Zimdahl, Phys. Rev. D 88, $103522(2013)$
31. H. Velten, J. Wang, X. Meng, Phys. Rev. D 88, 123504 (2013)

32. K. Bamba, S.D. Odintsov, arXiv:1508.05451 [gr-qc]

33. K. Bamba, A.N. Makarenko, A.N. Myagki, S. Nojiri, S.D. Odintsov, Bounce cosmology from $\mathrm{F}(\mathrm{R})$ gravity and $\mathrm{F}(\mathrm{R})$ bigravity. J. Cosmol. Astropart. Phys. 01, 008 (2014)

34. A. Astashenok, Effective dark energy models and dark energy models with bounce in frames of $F(T)$ gravity (2011). arXiv: 1308.058

35. K. Bamba, A.N. Makarenko, A.N. Myagky, S.D. Odintsov, Bouncing cosmology in modified Gauss-Bonnet gravity. Phys. Lett. B 732, 349-355 (2014)

36. S.D. Odintsov, V.K. Oikonomou, Matter bounce loop quantum cosmology from F (R) gravity. Phys. Rev. D 90, 124083 (2014)

37. K. Bamba, A.N. Makarenko, A.N. Myagky, S.D. Odintsov, (2014). arXiv: 1411.3852

38. M.R. Setare, M.J.S. Houndjo, (2013). arXiv: 1203.1315v3

39. R.D. Boko, M.J.S. Houndjo, J. Tossa, interacting cosmological viscous fluid with little rip and pseudo rip singularities solutions in $f(R)$ gravity. Int. J. Geom. Methods Mod. Phys. 15(2), 1850028 (2018). https://doi.org/10.1142/S0219887818500287

40. I. Brevik, V.V. Obukhov, A.V. Timoshkin, Bounce universe induced by an inhomogeneous dark fluid coupled with dark matter. Mod. Phys. Lett. A 29, 1450078 (2014)

41. S. Myrzakul, R. Myrzakulov, L. Sebastiani, Inhomogeneous viscous fluids in FRW universe and finite-future time singularities. Astrophys. Space Sci. 350, 845-853 (2014)

42. R. Myrzakulov, L. Sebastiani, Bounce solutions in viscous fluid cosmology. Astrophys. Space Sci. 352, 281-288 (2014)

43. Y.-F. Cai, S.-H. Chen, J.B. Dent, S. Dutta, E.N. Saridakis, Matter bounce cosmology with the $f(T)$ gravity (2011). arXiv: $1104.4649 \mathrm{v} 2$

44. I.G. Salako, M.E. Rodrigues, A.V. Kpadonou, M.J.S. Houndjo, J. Tossa, $\Lambda C D M$ model in $f(T)$ gravity: reconstruction, thermodynamics and stability. JCAP 11, 060 (2013). https://doi.org/10.1088/ 1475-7516/2013/11/060. arXiv:1307.0730

45. M. Sharif, S. Azeem, Cosmological evolution for dark energy models in $f(T)$ gravity (2013). arXiv:1305.0739v2

46. T. Qiu, K. Tian, S. Bu, Perturbations of bounce inflation scenario from $f(T)$ modified gravity revisited (2008). arXiv:1810.04436v1

47. K.L. Mahanta, A.K. Biswal, A dark energy model with viable EoS parameter in self-creation theory of gravitation. Rom. J. Phys. 58(3-4), 239-246 (2013)

48. V.R. Chirde, S.H. Shekh, Quadratic equation of state with constant deceleration parameter in $f(R)$ gravity. J. Theor. Phys. Cryptogr. 13 (2017)

49. C. Eckart, The thermodynamics of irreversible processes. III. Relativistic theory of the siple fluid. Phys. Rev. 58, 919 (1940)

50. D. Pavon, B. Wang, Le Châtelier-Braun principle in cosmological physics. Gen. Relativ. Gravit. 41, 1-5 (2009)

51. L. Amendola, G. Carmargo Campos, Consequences of dark matterdark energy interaction on cosmological parameters derived from type Ia Supernovae data. Phys. Rev. D 75, 083506 (2007)

52. Z.K. Guo, N. Ohta, S. Tsujikawa, Probing the coupling between dark components of the universe. Phys. Rev. D 76, 023508 (2007)

53. H. Amirhashchi, (2014). arXiv:1403.5258.v1 [astro-ph.CO]

54. S. Myrzakul, R. Myrzakulov, L. Sebastiani, Bounce solutions in viscous fluids cosmology. Astrophys. Space Sci. 352, 281-288 (2014)

55. P.H. Frampton, K.J. Ludwick, S. Nojiri, S.D. Odintsov, R.J. Scherrer, Models for little rip dark energy. Phys. Lett. B 708, 204-211 (2012)

56. I. Brevik, A.V. Timoshkin, Inhomogeneous dark fluid and dark matter, leading to a bounce cosmology. Universe 1, 24-37 (2015). arXiv:1503.02916 [gr-qc] 